Pathologe 2007 · 28:131-136

DOI 10.1007/s00292-007-0892-z

Online publiziert: 3. Februar 2007

๑) Springer Medizin Verlag 2007

\author{
E. Bruder · W. A. Meier-Ruge \\ Institut für Pathologie, Universitätsspital Basel, Schweiz
}

\title{
Hypoganglionose als Ursache chronischer Obstipation
}

sondere bei jungen Patienten von großer Bedeutung $[8,17]$.

Die vorliegende Arbeit bietet eine Übersicht über die Morphologie der verschiedenen Formen der Hypoganglionose, beschreibt die diagnostischen Techniken und diskutiert mögliche ätiologische Faktoren.

\section{Definition und Unterformen}

Die Hypoganglionose ist definiert als Verminderung des Nervenzellgehaltes typischerweise des Kolons oder auch seltener des gesamten Gastrointestinaltrakts. Der normale Nervenzellgehalt von Ganglien des Plexus myentericus liegt bei durchschnittlich $15 \pm 4$ Nervenzellen pro Ganglion. Die morphometrische Analyse enzymhistochemisch aufgearbeiteter Resektate von Kindern mit einer Hypoganglionose konnte eine Verminderung der Ganglienfläche und der Zahl der Nervenzellen des Plexus myentericus um $55 \%$ belegen [21, 22].

Es lassen sich 3 Hauptformen unterscheiden:

- die meist kongenitale hypoplastische

Hypoganglionose und

- die oligoneuronale dysganglionäre

Hypoganglionose mit Manifestation im Kindesalter;

- die atrophische Hypoganglionose ist eine häufige Ursache einer „Slowtransit-Obstipation " im fortgeschrittenen Erwachsenenalter.

Allen Formen der Hypoganglionose gemeinsam ist ein herabgesetzter Parasym- pathikotonus mit einer stark verminderten Acetylcholinesterase-Aktivität.

\section{Hypoplastische Hypoganglionose}

Die Hypoganglionose als isolierte kongenitale Fehlbildung ist selten und macht etwa 5\% intestinaler neuronaler Fehlbildungen aus [14]. Sie wird zunehmend als Ursache intestinaler Transportstörungen erkannt [17].

Die kongenitale hypoplastische Hypoganglionose kommt in 60\% der Patienten in Assoziation mit M. Hirschsprung vor. Sie kann auch isoliert auftreten. Die mit M. Hirschsprung assoziierte hypoplastische Hypoganglionose erstreckt sich meist zwischen 3 und $30 \mathrm{~cm}$ proximal des aganglionären Segments und wird auch als hypoganglionäre Übergangszone bezeichnet [21, 22, 35].

Die hypoplastische Hypoganglionose ist durch eine verminderte $\mathrm{Zahl}$ von Ganglien mit einem geringen Nervenzellgehalt pro Ganglion charakterisiert. Die Ganglien enthalten im Vergleich zur Norm von durchschnittlich $15 \pm 4$ Nervenzellen pro Ganglion lediglich $6 \pm 1$ Nervenzellen. Zudem sind die Abstände zwischen den Ganglien des Plexus myentericus um ein Vielfaches erhöht: Während bei normalen Kontrollen die Abstände der Ganglien bei durchschnittlich $170 \mathrm{~mm}$ liegen, betragen sie bei der Hypoganglionose über $420 \mathrm{~mm}$ [22].

Die hypoplastische Hypoganglionose manifestiert sich sowohl im Bereich des Plexus myentericus als auch im Plexus submucosus. Eine eindeutige Diagno- ist daher in der differenzialdiagnostischen Abgrenzung vom M. Hirschsprung insbe- 


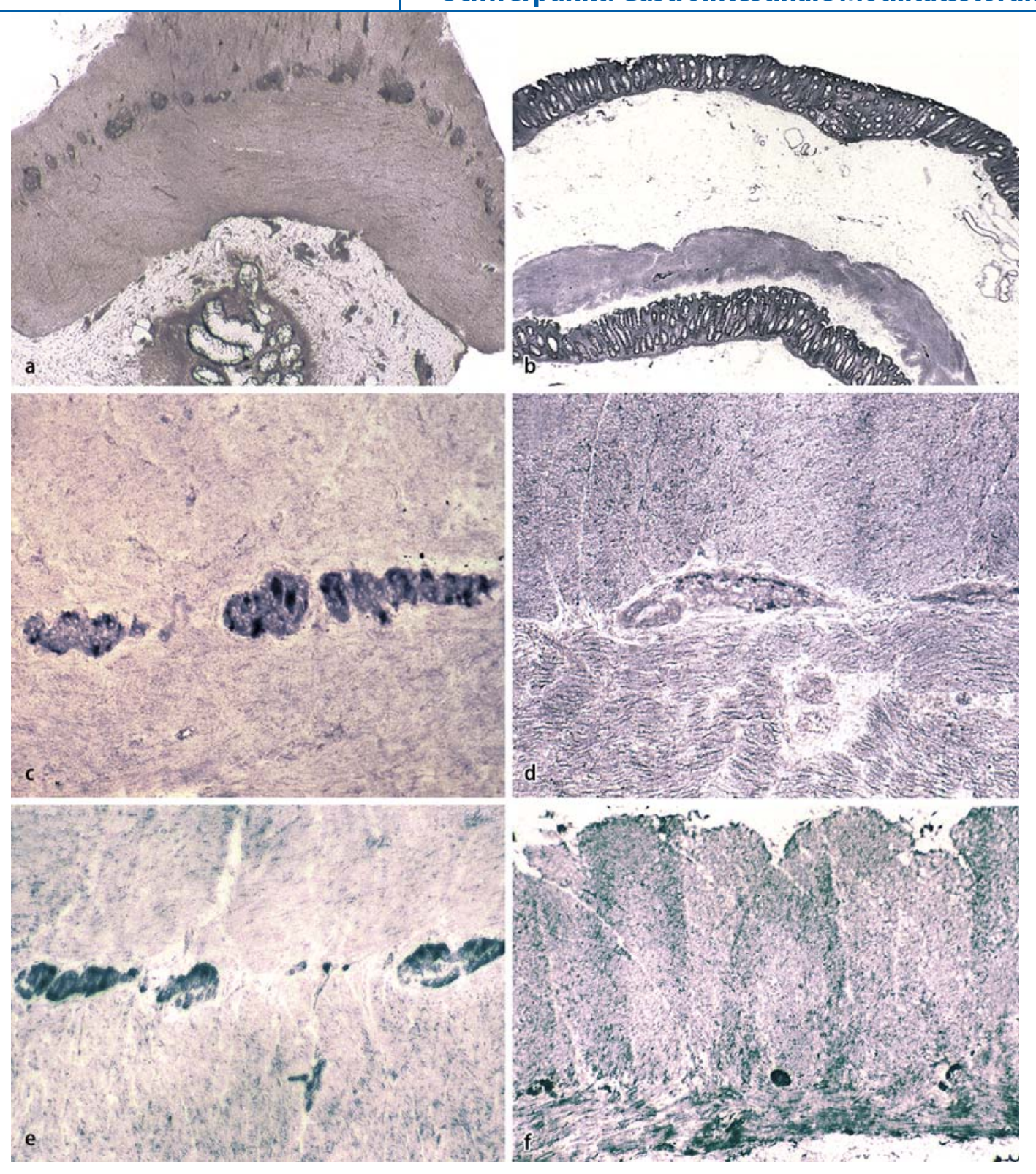

Abb. $1 \varangle$ Darstellung des Plexus myentericus mittels Enzymhistochemie [20]. a-d Laktatdehydrogenase: a Normaler Plexus myentericus (Vergr. 1:40). b Hypoplastische Hypoganglionose (Vergr. 1:16). c Normaler Plexus myentericus (Vergr. 1:180). d Oligoneuronale dysganglionäre Hypoganglionose (Vergr. 1:180). e,f Succinyldehydrogenase: e Normaler Plexus myentericus (Vergr. 1:180).

f Hypoplastische Hypoganglionose (Vergr. 1:120)

se lässt sich aber nur am Plexus myentericus stellen.

\section{Oligoneuronale dysganglionäre Hypoganglionose}

Die oligoneuronale dysganglionäre $\mathrm{Hy}$ poganglionose ist selten und $<1 \%$ der gastrointestinalen Innervationsstörungen aus. Während die hypoplastische Hypoganglionose in etwa 60\% der Patienten mit einem M. Hirschsprung assoziiert ist, kommt die oligoneuronale dysganglionäre Hypoganglionose im Allgemeinen isoliert vor $[16,17,24,25,35]$.

Im Gegensatz zur hypoplastischen Hypoganglionose ist die oligoneuronale dysganglionäre Hypoganglionose initial durch eine normale Anzahl von Ganglien des Plexus myentericus mit normalen interganglionären Abständen der myenterischen Ganglien charakterisiert. Hingegen zeichnen sich die Nervenzellen bei der oligoneuronalen dysganglionären $\mathrm{Hy}$ poganglionose durch ihre geringe Größe und einen spärlichen Zytoplasmaleib aus. Sie sind typischerweise meist in der Peripherie der Ganglien gelegen.

Zunächst ist die Zahl der Nervenzellen im einzelnen Ganglion normal. Der weitere Verlauf ist jedoch oft progredient. Nervenzellen und Ganglien gehen über Monate und Jahre zugrunde. Schließlich entwickelt sich eine hochgradige atrophische Hypoganglionose. Das morphologische Bild gleicht dann demjenigen ei- ner ausgeprägten hypoplastischen $\mathrm{Hy}$ poganglionose. Diese spezielle Form der progressiven Hypoganglionose dehnt sich auch auf den Dünndarm aus.

\section{Atrophische Hypoganglionose des Erwachsenen}

Im Gegensatz zur hypoplastischen Hypoganglionose ist die atrophische Form ein sekundäres Krankheitsbild. In bis zu 55\% der erwachsenen Patienten mit therapieresistenter chronischer Obstipation kann histologisch eine atrophische Hypoganglionose nachgewiesen werden [9, 23]. Quantitative morphometrische Analysen konnten an immunhistochemisch untersuchten Darmresektaten zeigen, dass auch 
bei erwachsenen Patienten mit „slow transit constipation" sowohl die Zahl der Nervenzellen pro Ganglion, als auch die Gesamtzahl der Ganglien im Plexus myentericus signifikant vermindert ist [35].

\section{Interstitielle \\ Schrittmacherzellen Cajal}

Die Zahl der Cajal-Zellen in der Muscularis propria ist bei Patienten mit Hypoganglionose als vermindert beschrieben worden $[29,35,36,38]$. Den gastrointestinalen „pacemaker cells“ nach Cajal kommt eine essenzielle Aufgabe bei der neuromuskulären Signalübermittlung zu. Verteilung und Anzahl der gastrointestinalen Pacemaker-Zellen bei gastrointestinalen Motilitätsstörungen sind gegenwärtig Gegenstand intensiver Forschung.

\section{Diagnostik}

Bei Patienten mit chronischer therapieresistenter Obstipation ist nach ausführlicher klinischer und radiologischer Abklärung eine Biopsie der Muscularis propria und damit des Plexus myentericus zur Untersuchung des enteralen Nervensystems indiziert.

Die quantitative Einschätzung des bei der Hypoganglionose verminderten Ganglien- und Nervenzellbesatzes des Plexus myentericus gelingt am besten mit einer selektiven Anfärbung der Nervenzellen in den enzymhistochemischen Laktatdehydrogenase-, Succinatdehydrogenase- und Nitrit-Oxid-SynthetaseReaktionen (• Abb. 1 a-f; [18, 19, 20]). Zur Sicherung der Diagnose einer Hypoganglionose sind daher native Biopsien der Muscularis propria des betroffenen Darmabschnitts erforderlich. Biopsien der Muscularis propria intraperitonealer Darmabschnitte können mittels einer minimal-invasiven laparoskopischen Technik entnommen werden und bergen in erfahrenen Händen kein wesentlich höheres Komplikationsrisiko als endoskopisch gewonnene Schleimhautbiopsien.

Die Qualität des Plexus myentericus im Bereich der geplanten Anastomosenzone kann auch intraoperativ am nativen Gefrierschnitt innerhalb von etwa 8-15 min mittels einer enzymhistochemischen Laktatdehydrogenase-Reak-

Pathologe 2007 · 28:131-136 DOI 10.1007/s00292-007-0892-z

(C) Springer Medizin Verlag 2007

\section{E. Bruder $\cdot$ W. A. Meier-Ruge Hypoganglionose als Ursache chronischer Obstipation}

\section{Zusammenfassung}

Die mit einer chronischen therapieresistenten Obstipation verbundene Hypoganglionose macht 3-5\% der bioptisch abzuklärenden gastrointestinalen Innervationsstörungen im Kindes- und Erwachsenenalter aus. Sie kann wie der M. Hirschsprung zu einem Megakolon führen und muss als wichtige Differenzialdiagnose berücksichtigt werden. Es lassen sich 3 Hauptformen der Hypoganglionose unterscheiden: Die kongenitale hypoplastische Hypoganglionose tritt vorwiegend beim klassischen M. Hirschsprung proximal des aganglionären Segments auf. Sie zeigt kleine zellarme Ganglien mit großen interganglionären Abständen. Die oligoneuronale dysganglionäre Hypoganglionose manifestiert sich im Kindesalter und ist charakterisiert durch eine zunächst normale Zahl hypoplastischer Nervenzellen normal großer Ganglien des Plexus myentericus. Diese Form der Hypoganglionose kann progressiv ver- laufen und in eine atrophische Hypoganglionose münden, die im Erwachsenenalter ein der Hirschsprung-assoziierten hypoplastischen Hypoganglionose ähnliches morphologisches Bild zeigt. Alle Formen äußern sich in einer verminderten Acetylcholinesterase-Aktivität der Nervenfasern der Muscularis propria. Über die Ätiologie der Hypoganglionose ist wenig bekannt: Bei den Hirschsprung assoziierten Formen sind vereinzelt Mutationen im RET- und GDNF-Gen nachgewiesen worden. Im heterozygoten $\mathrm{GDNF}^{+-}$-Tiermodell führt die GDNF-Mutation zur Hypoganglionose, was bei der isolierten Hypoganglionose des Menschen noch zu belegen ist.

\section{Schlüsselwörter}

Hypoganglionose - Chronische Obstipation . Enzymhistochemie - Plexus myentericus . Genetik

\section{Hypoganglionosis as a cause of chronic constipation}

\section{Abstract}

Hypoganglionosis comprises 3-5\% of gastrointestinal innervation defects which are connected to therapy-resistant chronic constipation in children and adults. Similar to Hirschsprung's disease, hypoganglionosis may be complicated by megacolon formation and must be considered in the differential diagnosis. Three main subtypes may be distinguished: congenital hypoplastic hypoganglionosis occurs predominantly in Hirschsprung's disease proximal to the aganglionic segment and consists of small paucicellular ganglia with increased interganglionic distances. Oligoneuronal dysganglionic hypoganglionosis manifests in childhood. Initially, myenteric ganglia are of normal size and have normal interganglionic spacing and normal neuronal content. However, nerve cells are hypoplastic and ganglia undergo progressive nerve cell loss. This type of hypoganglionosis may progress into atrophic hypoganglionosis, which shows a morphology similar to hypoplastic hypoganglionosis. All subtypes of hypoganglionosis result in decreased acetylcholinesterase activity in the nerve fiber network of the muscularis propria. The pathogenesis of hypoganglionosis is still poorly understood. In Hirschsprung associated hypoganglionosis, mutations in the RET and GDNF-genes have been found. Despite a heterozygote GDNF+/- animal model for hypoganglionosis, no GDNF mutations have so far been demonstrated in human Hirschsprung independent, isolated hypoganglionosis.

\section{Keywords}

Hypoganglionosis - Chronic constipation . Enzyme histochemistry - Myenteric plexus . Genetics 


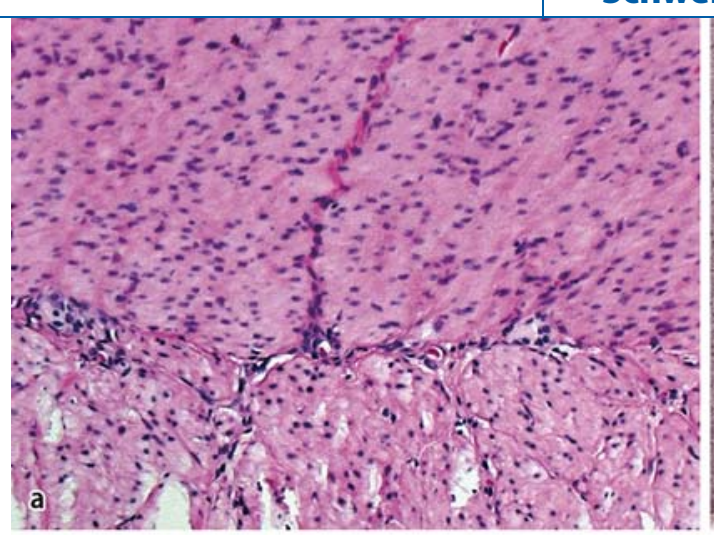

\section{Schwerpunkt: Gastrointestinale Motilitätsstörungen}
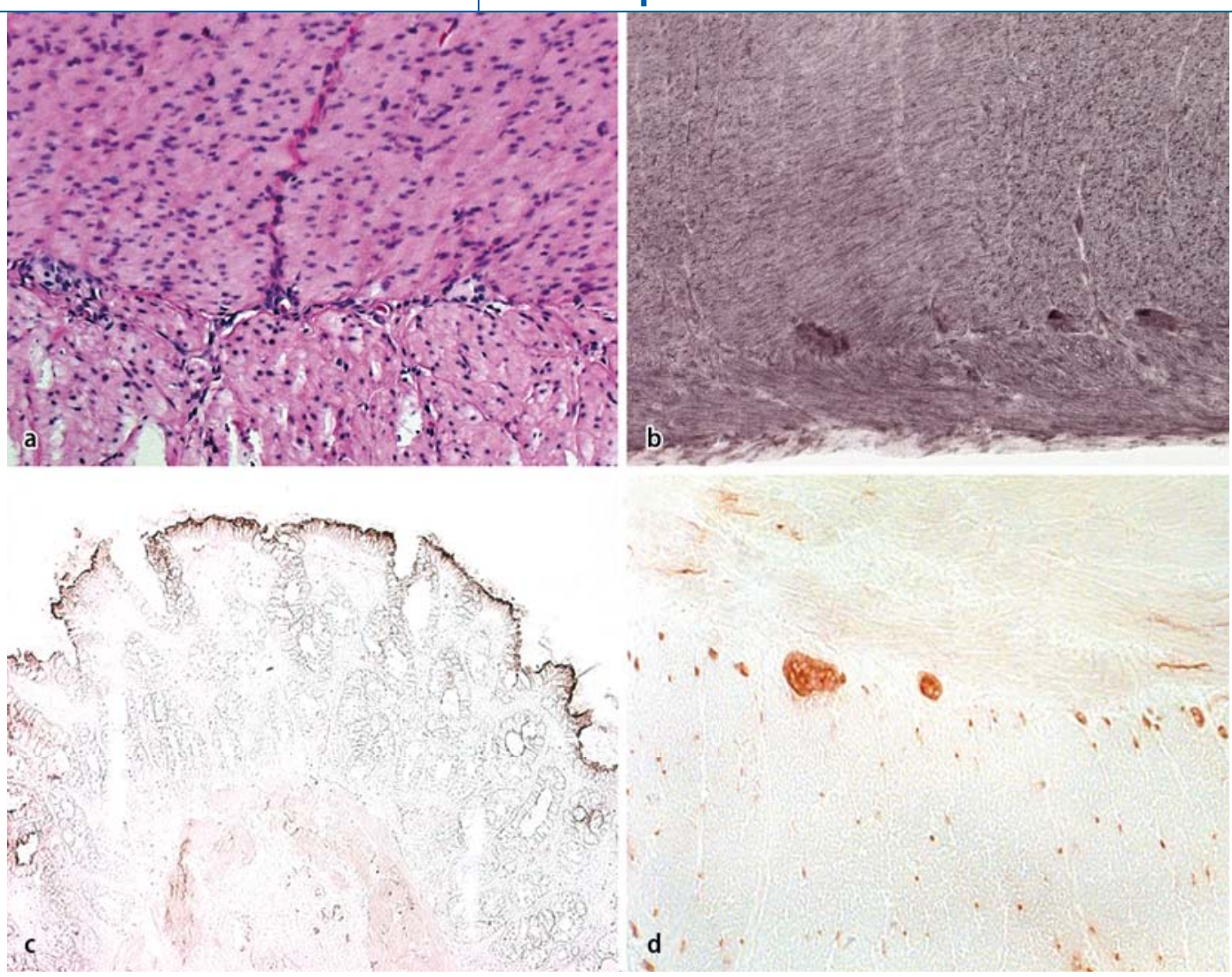

Abb. $2<$ Hypoplastische Hypoganglionose des Plexus myentericus. a HE-Färbung (Vergr. 1:180). b Laktatdehydrogenase-Enzymhistochemie (Vergr. 1:180). c Verminderung der Acetylcholinesterase-Aktivität der Nervenfasernetze der Muscularis mucosae (Acetylcholinesterase-Enzymhistochemie, Vergr. 1:180). d Verminderte Acetylcholinesterase-Aktivität der Nervenfasernetze der Muscularis propria (Acetylcholinesterase-Enzymhistochemie, Vergr. 1:180)

tion überprüft werden. Bei einer einzeitigen Operation erlaubt diese intraoperative Schnellschnitttechnik eine wesentlich präzisere Platzierung der definitiven Anastomose, als dies inspektorisch oder radiologisch möglich ist [20].

Alle Formen der Hypoganglionose gehen charakteristischerweise mit einer verminderten Acetylcholinesterase-Aktivität der Nervenfasernetze der Muscularis propria und der Muscularis mucosae einher ( $\bullet$ Abb. 2 a-d). Diese korreliert mit der verminderten peristaltischen Transportleistung im biopsierten Darmsegment und wird durch die enzymhistochemische Darstellung der Acetylcholinesterase-Aktivität der cholinergen Nervenfasernetze der Muscularis propria erfasst $[11,15,20]$.

Ein erniedrigter Parasympathikotonus der Nervenfasernetze der Muscularis mucosae kann zusammen mit einem spärlich entwickelten Plexus submucosus in einer Schleimhautbiopsie auf eine mögliche Hypoganglionose des Plexus myentericus hinweisen, doch ist für die beweisende Diagnose eine Biopsie der Mucularis propria mit Anteilen des Plexus myentericus erforderlich $[20,26]$.
Zwar lassen sich an formalinfixierten und paraffineingebetteten Biopsien der Muscularis propria die Nervenzellen der Ganglien des Plexus myentericus auch immunhistochemisch mittels Cathepsin D und PGP 9.5 gut darstellen, doch ist die Sensitivität der enzymhistochemischen Reaktionen höher, und es fehlt der Immunhistochemie die Möglichkeit der wichtigen funktionellen Einschätzung des Parasympathikotonus.

\section{Ätiologie und Genetik}

Die Ursache der Hypoganglionose ist noch nicht abschließend geklärt. Es ist davon auszugehen, dass nicht eine einheitliche Ursache wirksam ist, sondern eine Reihe von verschiedenen Faktoren zusammenwirken.

Das enterale Nervensystem leitet sich aus Stammzellen der Neuralleiste ab [5]. Ein Durchbruch in der Erforschung der Entwicklung des enteralen Nervensystems erfolgte mit der Möglichkeit der Isolierung von Neuralleistenzellen. An isolierten Neuralleistenzellen kann der Effekt von Wachstumsfaktoren auf die Entwicklung enteraler Neurone oder Glia untersucht werden. Neurotrophin 3 fördert die Entwicklung enterischer Nervenzellen [5]. TRK-C- und NT-3-Knockout Mäuse zeigen eine Reduktion der Gesamtzahl von enterischen Neuronen im proximalen Ileum. Dieses Modell unterstützt die Annahme, dass eine Hypoganglionose wahrscheinlich einem Mangel an neurotrophischen Faktoren zuzuschreiben ist.

Die „Bone-morphogenetic-Proteine" (BMP) 2 und 4 und deren Rezeptor BMPRII werden während der Darmentwicklung stark exprimiert. Ist die Aktivität von BMP gehemmt, resultiert eine Hypoganglionose [6]. Auch bei der Genese der kongenitalen intestinalen Motilitätsstörungen von M. Hirschsprung und intestinaler neuronaler Dysplasie (IND) B könnte der BMP-Signalkaskade eine wichtige Rolle zukommen [18]. Im Tiermodell findet sich außerdem bei der heterozygoten "glial derived neurotrophic factor-“ $(G D N F-)^{+/-}$Maus eine langstreckige Hypoganglionose des Darms [34]. Bei der Hypoganglionose des Menschen ergaben Untersuchungen bei 5 Kindern bisher keine GDNF-Mutationen [9].

Eine Hypoganglionose kann proximal des aganglionären Segments einer Hirschsprung-Erkrankung oder assoziiert mit IND B vorkommen $[18,19]$. Auch 
in Tiermodellen sind Übergangsformen zwischen Hypo- und Aganglionose beobachtet worden [1]. Patienten mit einem M. Hirschsprung besitzen in $15-45 \%$ eine Keimbahnmutation des RET-Gens und einige dieser Patienten wiederum assoziierte Mutationen des RET-Liganden GDNF $[4,10,27,28,30]$. Bei Patienten mit isolierter Hypoganglionose konnten vereinzelt einzelne Basensubstitutionen der Tyrosinkinase-Domäne des RET-Gens nachgewiesen werden, die als „stille Mutationen" klassifiziert wurden [9]. Diese molekulargenetischen Befunde legen nahe, dass RET und GDNF in der Pathogenese der Hypoganglionose des Menschen eher eine geringe Rolle zukommt.

Erworbene Formen einer atrophischen Hypoganglionose werden bei Erwachsenen beschrieben [24]. Die Verminderung der Zahl der Neuronen wird verschiedenen Ursachen zugeschrieben wie Hypoxie, toxischen, autoimmunen, viralen, idiopathisch degenerativen oder metabolischen Prozessen [12, 13, 25, 32, 37].

\section{Therapie}

Bisher stehen für die manifeste, klinisch symptomatische und konservativ therapieresistente Hypoganglionose nur chirurgische Therapiemöglichkeiten zur Verfügung. Da die funktionsschwache Peristaltik im hypoganglionären Darmabschnitt keinen effizienten Transport des Darminhalts gewährleisten kann, sollte die hypoganglionäre Übergangszone möglichst vollständig mit dem aganglionären Darmsegment reseziert werden, um eine normale und wirkungsvolle Innervation proximal der Anastomose zu ermöglichen [2, 33].

Bleibt ein kurzes hypoganglionäres Restsegment proximal einer Aganglionose zurück, resultiert oft keine relevante Obstipation. Ist das verbleibende hypoganglionäre Segment jedoch lang, so kommt es bei der Hirschsprung-assoziierten Hypoganglionose in der Mehrzahl der Patienten zum postoperativen Rezidiv der chronischen Obstipation, sodass das hypoganglionäre Segment nachreseziert werden muss $[2,33]$.

Es ist zu erwarten, dass mit zunehmender Kenntnis der Migrations- und Reifungsfaktoren des enteralen Nervensystems und dessen Stammzellen in $\mathrm{Zu}$ - kunft eine medikamentöse, genetische oder Stammzelltherapie auch der Hypoganglionose möglich werden könnte. Der pharmakotherapeutische Ersatz von neurotrophischen Faktoren könnte in der $\mathrm{Zu}$ kunft an Bedeutung gewinnen und möglicherweise an die Stelle chirurgischer Resektion treten.

\section{Fazit und Ausblick}

\section{Die Hypoganglionose ist verantwortlich} für etwa 5\% der kongenitalen Motilitätsstörungen und findet sich bei bis zu $55 \%$ biopsierter Patienten mit „slow transit constipation". Sie ist definiert als Verminderung des Nervenzellbesatzes des Plexus myentericus um etwa 50\%. Die zuverlässige Diagnose erfordert Biopsien der Muscularis propria mit Anteilen des Plexus myentericus. Biopsien lediglich der Schleimhaut des betroffenen Darmabschnitts sind zur Diagnose einer Hypoganglionose nicht ausreichend. Die selektive quantitative Darstellung der Nervenzellen des Plexus myentericus gelingt am besten an nativen Biopsien mittels der enzymhistochemischen Laktatdehydrogenase-Reaktion und korreliert mit einer verminderten AcetylcholinesteraseAktivität der Nervenfasernetze der Muscularis propria. Zwar ist die Markierung der Ganglienzellen auch immunhistochemisch mit dem Antikörper gegen Cathepsin D möglich, doch lässt sich der verminderte Parasympathikotonus nur enzymhistochemisch erfassen (fertige enzymhistochemische Kits: Districhem Oberwil, CH-4104 Oberwil, E-Mail: districhem@ bluewin.ch oder Bio-Optica, Via San Faustina, I-20134 Milano: http://www.biooptica). Therapeutisch bleibt gegenwärtig nach erfolgloser konservativer Behandlung nur die chirurgische Resektion.

\section{Korrespondierender Autor}

Prof. Dr. W. A. Meier-Ruge

Institut für Pathologie, Universitätsspital Basel Schönbeinstraße 40, 4031 Basel, Schweiz meier-ruge@bluewin.ch

Interessenkonflikt. Es besteht kein Interessenkonflikt. Der korrespondierende Autor versichert, dass keine Verbindungen mit einer Firma, deren Produkt in dem Artikel genannt ist, oder einer Firma, die ein Konkurrenzprodukt vertreibt, bestehen. Die Präsentation des Themas ist unabhängig und die Darstellung der Inhalte produktneutral.

\section{Literatur}

1. Cass DT (2000) Animal models of aganglionosis. In: Holschneider AM, Puri P (eds) Hirschsprung's disease and allied disorders. Harwood Academic Publishers, Amsterdam, pp 59-68

2. Farrugia MK, Alexander N, Clarke $\mathrm{S}$ et al. (2003) Does transitional zone pull-through in Hirschsprung's disease imply a poor prognosis? J Pediatr Surg 38: 1766-1769

3. Faussone-Pellegrini MS, Infantino A, Matini P et al. (1999) Neuronal anomalies and normal muscle morphology at the hypomotile ileocecocolonic region of patients affected by idiopathic chronic constipation. Histol Histopathol 14: 1119-1134

4. Gabriel SB, Salomon R, Pelet A et al. (2002) Segregation at three loci explains familial and population risk in Hirschsprung disease. Nat Genet 31: 89-93

5. Gershon MD, Ratcliffe EM (2004) Developmental biology of the enteric nervous system: pathogenesis of Hirschsprung's disease and other congenital dysmotilities. Semin Pediatr Surg 13: 224-235

6. Goldstein AM, Brewer KC, Doyle AM et al. (2005) $B M P$ signaling is necessary for neural crest cell migration and ganglion formation in the enteric nervous system. Mech Dev 122: 821-833
Hier steht eine Anzeige. Springer 
7. Holschneider AM, Meier-Ruge W, Ure BM (1994) Hirschsprung's disease and allied disorders - a review. Eur J Pediatr Surg 4: 260-266

8. Ikeda K, Goto S, Nagasaki A, Taguchi T (1988) Hypogenesis of intestinal ganglion cells: a rare cause of intestinal obstruction simulating aganglionosis. Z Kinderchir 43: 52-53

9. Inoue K, Shimotake T, Tomiyama H, Iwai N (2001) Mutational analysis of the RET and GDNF gene in children with hypoganglionosis. Eur J Pediatr Surg 11: 120-123

10. Kapur RP, Gershon MD, Milla PJ, Pachnis V (2004) The influence of Hox genes and three intercellular signalling pathways on enteric neuromuscular development. Neurogastroenterol Motil 16 Suppl 1: 8-13

11. Kobayashi H, Li Z, Yamataka A et al. (2002) Acetylcholinesterase distribution and refractory constipation - a new criterion for diagnosis and management. Pediatr Surg Int 18: 349-353

12. Lennon VA, Sas DF, Busk MF et al. (1991) Enteric neuronal autoantibodies in pseudoobstruction with small-cell lung carcinoma. Gastroenterology 100: 137-142

13. Matsui T, Iwashita A, lida M et al. (1987) Acquired pseudoobstruction of the colon due to segmental hypoganglionosis. Gastrointest Radiol 12: 262-264

14. Meier-Ruge W (1992) Epidemiology of congenital innervation defects of the distal colon. Virchows Arch A Pathol Anat Histopathol 420: 171-177

15. Meier-Ruge W, Lutterbeck PM, Herzog B et al. (1972) Acetylcholinesterase activity in suction biopsies of the rectum in the diagnosis of Hirschsprung's disease. J Pediatr Surg 7: 11-17

16. Meier-Ruge WA (2000) Comparative investigation of hypoganglionosis in the gut of children and adults with chronic constipation. In: Krammer H-J, Singer MV (eds) Neurogastroenterology: from the basics to the clinics. Kluwer Acac Publ, Dordrecht Niederland, pp 493-495

17. Meier-Ruge WA (2000) Histological diagnosis and differential diagnosis. In: Holschneider AM, Puri P (eds) Hirschsprung's disease and allied disorders. Harwood, Amsterdam, pp 252-265

18. Meier-Ruge WA, Ammann K, Bruder E et al. (2004) Updated results on intestinal neuronal dysplasia (IND B). Eur J Pediatr Surg 14: 384-391

19. Meier-Ruge WA, Bronnimann PB, Gambazzi F et al. (1995) Histopathological criteria for intestinal neuronal dysplasia of the submucosal plexus (type B). Virchows Arch 426: 549-556

20. Meier-Ruge WA, Bruder E (2005) Pathology of chronic constipation in pediatric and adult coloproctology. Pathobiology 72: 1-102

21. Meier-Ruge WA, Brunner LA (2001) Morphometric assessment of Hirschsprung's disease: associated hypoganglionosis of the colonic myenteric plexus. Pediatr Dev Pathol 4: 53-61

22. Meier-Ruge WA, Brunner LA, Engert J et al. (1999) A correlative morphometric and clinical investigation of hypoganglionosis of the colon in children. Eur J Pediatr Surg 9: 67-74

23. Munakata K, Fukuzawa M, Nemoto N (2002) Histologic criteria for the diagnosis of allied diseases of Hirschsprung's disease in adults. Eur J Pediatr Surg 12: 186-191

24. Munakata K HA (2000) Megacolon in Adults. In: Holschneider AM, Puri P (eds) Hirschsprung's disease and allied disorders. Harwood, Amsterdam, pp 175-183
25. Munakata K NS, Yasumuro S, Nagai N, Morita K (1991) Intestinal obstruction caused by secondary degeneration of intramural plexus: a case report. In: Yoshikawa M et al. (eds) New trends in autonomic nervous system research. Excerpta Medica, Amsterdam London New York Tokyo, pp 287-288

26. Coerdt W, Müntefering $\mathrm{H}$, Rastorguev $\mathrm{E}$, Gerein V (2004) Kongenitale Innervationsstörungen des Kolon. Der Pathologe 25: 292-298

27. Newgreen D, Young HM (2002) Enteric nervous system: development and developmental disturbances - part 1. Pediatr Dev Pathol 5: 224-247

28. Newgreen D, Young HM (2002) Enteric nervous system: development and developmental disturbances - part 2. Pediatr Dev Pathol 5: 329-349

29. Park SH, Min H, Chi JG et al. (2005) Immunohistochemical studies of pediatric intestinal pseudoobstruction: bcl2, a valuable biomarker to detect immature enteric ganglion cells. Am J Surg Pathol 29: 1017-1024

30. Passarge E (2002) Dissecting Hirschsprung disease. Nat Genet 31: 11-12

31. Qualman SJ, Murray R (1994) Aganglionosis and related disorders. Hum Pathol 25: 1141-1149

32. Schobinger-Clement $\mathrm{S}$, Gerber HA, Stallmach $T$ (1999) Autoaggressive inflammation of the myenteric plexus resulting in intestinal pseudoobstruction. Am J Surg Pathol 23: 602-606

33. Schulten D, Holschneider AM, Meier-Ruge W (2000) Proximal segment histology of resected bowel in Hirschsprung's disease predicts postoperative bowel function. Eur J Pediatr Surg 10: 378-381

34. Shen L, Pichel JG, Mayeli T et al. (2002) Gdnf haploinsufficiency causes Hirschsprung-like intestinal obstruction and early-onset lethality in mice. Am J Hum Genet 70: 435-447

35. Wedel T, Roblick UJ, Ott V et al. (2002) Oligoneurona hypoganglionosis in patients with idiopathic slowtransit constipation. Dis Colon Rectum 45: 54-62

36. Wedel T, Spiegler J, Soellner S et al. (2002) Enteric nerves and interstitial cells of Cajal are altered in patients with slow-transit constipation and megacolon. Gastroenterology 123: 1459-1467

37. Wedel T, Tafazzoli K, Sollner S et al. (2003) Mitochondrial myopathy (complex I deficiency) associated with chronic intestinal pseudo-obstruction. Eur J Pediatr Surg 13: 201-205

38. Yu CS, Kim HC, Hong HK et al. (2002) Evaluation of myenteric ganglion cells and interstitial cells of $\mathrm{Ca}-$ jal in patients with chronic idiopathic constipation. Int J Colorectal Dis 17: 253-258

\section{www.Der Pathologe.de Kongressanmeldung nur noch online}

Nutzen Sie www.DerPathologe.de, um Ihre Veranstaltungen in den Springer Fachzeitschriften Medizin/Psychologie und dem dazugehörigen Online-Angebot kostenlos anzukündigen.

Bitte beachten Sie, dass wir nur noch Ankündigungen berücksichtigen werden, die uns online erreichen.

Und so geht's:

- Klicken Sie auf www.DerPathologe.de den Button „Kongresse" an

- Wählen Sie dort „Veranstaltung anmelden"

- Geben Sie nun Ihre Veranstaltung in die Eingabemaske ein

- Wählen Sie die Zeitschriften aus, in denen Ihre Ankündigung erscheinen soll

- Schicken Sie das ausgefüllte Formular online an die Verlagsredaktion

In den Print-Ausgaben unserer Zeitschriften bieten wir unseren Lesern anschließend in der Rubrik "Termine" eine Auswahl aktueller Veranstaltungen. Eine erheblich umfangreichere Auflistung steht online zur Verfügung. Die Redaktion behält sich eine Auswahl von Ankündigungen vor.

Für Veranstaltungshinweise in Form von Anzeigen können Sie sich jederzeit an unsere Anzeigenabteilung wenden (anzeigen@springer-sbm.com)

Wir freuen uns auf Ihre Ankündigungen!

Mit freundlichen Grüßen

Ihre Redaktion

Fachzeitschriften Medizin/Psychologie

www.DerPathologe.de 